\title{
Controlling morphology and crystal structure of tungsten nitride nanomaterials
}

Olivia Wenzel ${ }^{1}$, Viktor Rein ${ }^{2}$, Milena Hugenschmidt ${ }^{3}$, Claus Feldmann ${ }^{4}$ and Dagmar Gerthsen ${ }^{5}$

${ }^{1}$ Karlsruhe Institute of Technology, Laboratory for Electron Microscopy, Karlsruhe, Baden-Wurttemberg, Germany, ${ }^{2}$ Karlsruhe Institute of Technology, Institute of Inorganic Chemistry, Baden-Wurttemberg, Germany, ${ }^{3}$ Karlsruhe Institute of Technology, Laboratory for Electron Microscopy, Karlsruhe, Germany, ${ }^{4}$ Karlsruhe Institute of Technology, Institute of Inorganic Chemistry, Karlsruhe, Baden-Wurttemberg, Germany, ${ }^{5}$ Laboratorium für Elektronenmikroskopie, Karlsruher Institut für Technologie (KIT), Engesserstr. 7, 76131 Karlsruhe, Germany, United States

Tungsten nitride (WNx) is a popular compound in microelectronics due to its favorable material properties such as high melting point, tremendous hardness and good electrical conductivity [1]. Furthermore, nanoscaled WNx is of interest in the field of catalysis, where it might reach catalytic properties comparable to that of noble metals in various redox-reactions and oxygen-reduction reactions in fuel cells [2]-[7]. However, WNx nanomaterials have received less attention compared to other material classes mainly due to difficulties in production. Conventional synthesis routes require high temperatures, which is in opposition to the desired nanoscaled geometry $[8,9]$. Moreover, WNx nanomaterials can easily react with oxygen and water in the atmosphere thereby deteriorating the material. These problems motivate the development of novel syntheses approaches to obtain homogeneous and high-purity nitride nanomaterials.

This work presents two types of WNx nanomaterials, one composed of nanocrystalline aggregates and the other made up of nanosheets. Both nanomaterials were synthesized with an alkylamide-based synthesis, which avoids all use of oxygen-bearing compounds and allows manipulation of geometry and morphology. This control is enabled by long-chain amines that form a lyotropic or lamellar phase depending on the surfactant concentration. The structural and chemical properties of the $\mathrm{WNx}$ nanomaterials were studied in detail by different electron microscopic techniques in combination with electron spectroscopic analyses. Material synthesis and sample preparation for transmission electron microscopy (TEM) were performed in an argon atmosphere (Schlenk line and glove-box). The samples were transferred into the electron microscope with a TEM transfer holder to protect the material from oxidation and hydrolysis.

The nanocrystalline WNx, as shown by HAADF-STEM images in Fig.1(a,b) consists of 0.8 - $7.6 \mathrm{~nm}$ small crystallites, which are loosely aggregated, thereby forming a nanoporous substructure. These small crystallites have a cubic Fm-3m crystal structure with a composition of WN0.7, which is compatible with the composition range of the cubic $\square$-WNx phase [10].

If a lamellar phase is formed due to higher surfactant concentration, the synthesis yields WNx nanosheets. Scanning TEM (STEM) with a high-angle annular dark-field detector (HAADF-STEM) as well as secondary-electron scanning electron microscopy (SE-SEM) imaging show the nannosheets to have lateral dimensions of up to $500 \mathrm{~nm}$ and a mean thickness of $2.1 \pm 1.1 \mathrm{~nm}$ (c.f. Fig.1(c,d)). Compared to the nanocrystalline WNx, the nanosheets are richer in nitrogen with a compositional range of WN1.7-3.7 and occur in the hexagonal crystal structure of the $\delta$ WNx phase [10]. The nanosheets are often stacked on top of one another with frequent rotations of $4-6^{\circ}$ around the c axis of the hexagonal crystal structure, which produces characteristic and complex Moiré patterns as show in the HRTEM image Fig.2(d,e). With these rotations between the stacked nanosheets they form commensurate interface structures. High stacking-fault densities and signs of nanotwins can be repeatedly observed in nanosheets. These defects appear as striped contrast in TEM images such as in the blue rectangle in Fig.2(a,b). Further V-shaped defects parallel to hexagonal a1 and a2 axes are present in Fig.2(b) and can be interpreted as nanotwins. 
This study shows that WNx nanomaterials with strikingly different morphologies were successfully synthesized by tuning the long-chain amine concentration in the alkylamide-based synthesis. Looking ahead, this technique should also be applicable to other transition metal nitrides, which opens up the possibility of a still yet unexplored new class nanoscaled materials.
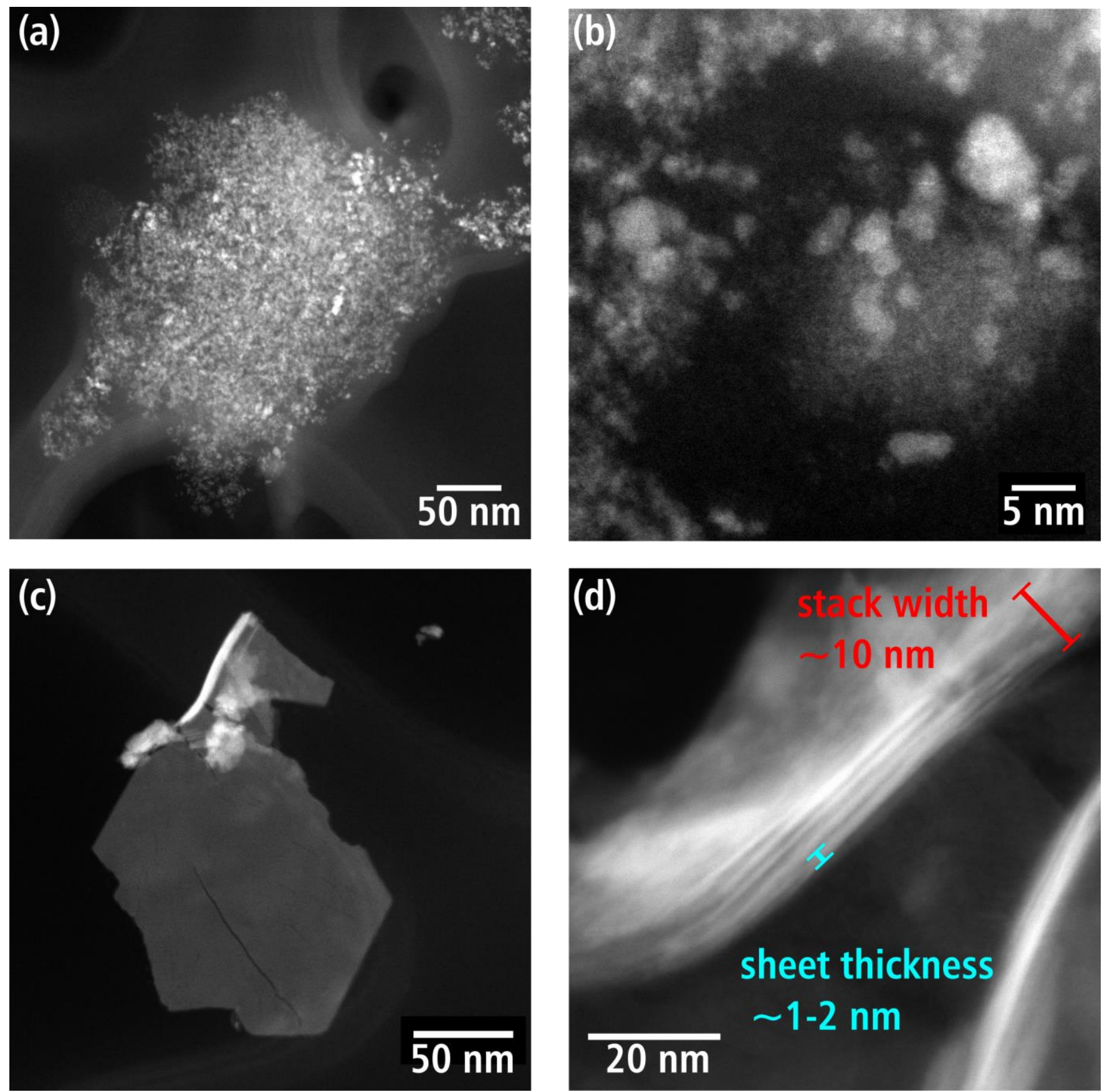

Figure 1. (a) HAADF-STEM overview image of a nanocrystalline $\mathrm{WNx}$ and (b) image taken at larger magnification. (c) HAADF-STEM overview of a WNx nanosheet. Some sheets are stacked on top of one another as shown in (d). 

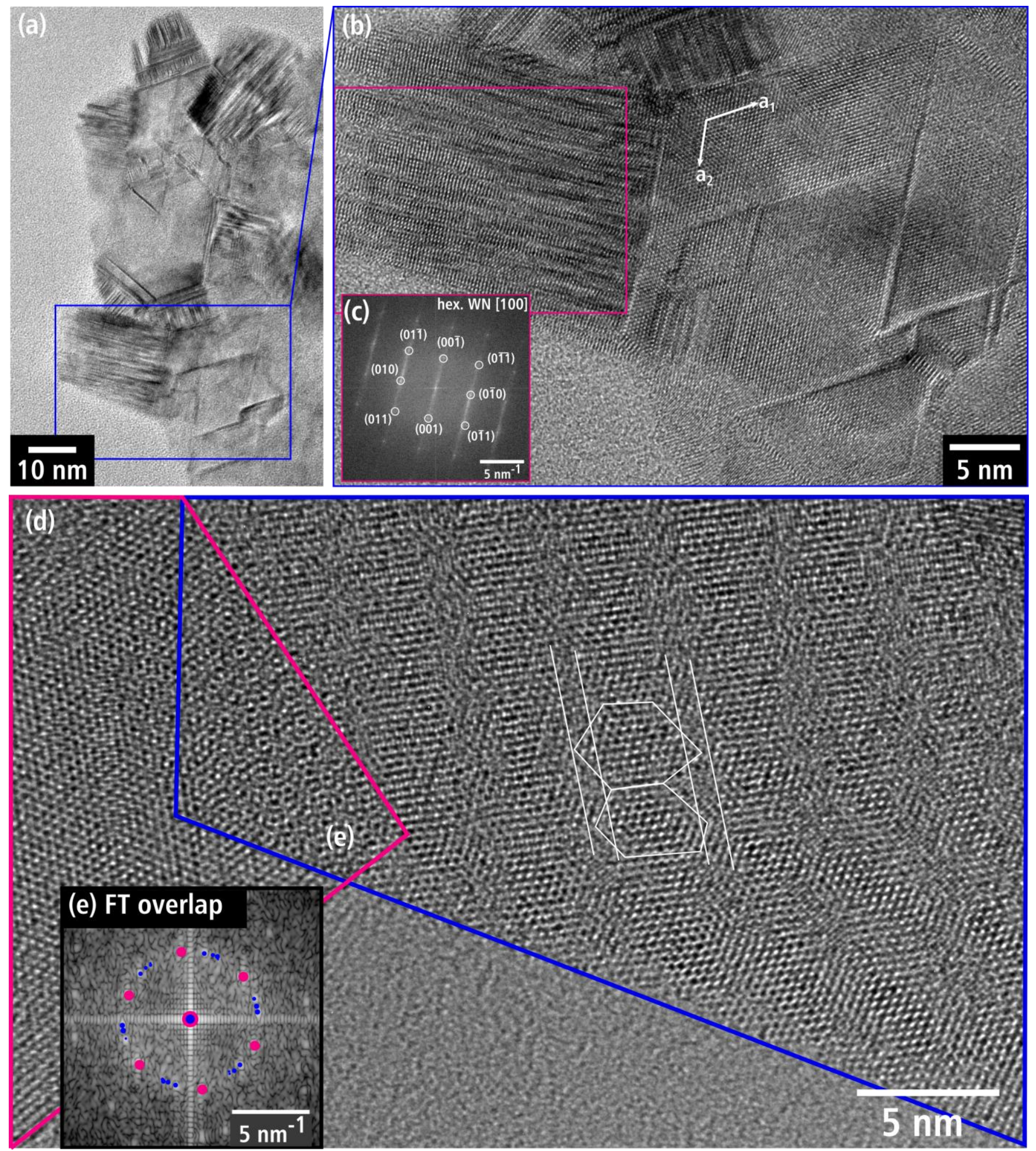

Figure 2. (a) HRTEM image of agglomerated WNx nanosheets. (b) Magnification of the region marked in (a) by a blue rectangle. The region marked with a pink frame in (b) shows a striped contrast induced by stacking faults as further illustrated in the corresponding FT image (c). (d) HRTEM image of an edge region of several stacked sheets shows a complex moiré pattern (blue) and a single sheet (pink). (e) shows the FT image of the overlap region. 


\section{References}

[1] Y. T. Kim, C. W. Lee, C. S. Kwon, S. K. Min, and S. K. Park, "Characteristics of amorphous tungsten nitride diffusion barrier for metal-organic chemical vapor deposited $\mathrm{Cu}$ metallization," in 1994 International Electron Devices and Materials Symposium, EDMS 1994, 1994, pp. 8-6-21-8-6-24.

[2] S. T. Oyama, "Preparation and catalytic properties of transition metal carbides and nitrides," Catal. Today, vol. 15, no. 2, pp. 179-200, Jun. 1992.

[3] J. S. J. Hargreaves, "Heterogeneous catalysis with metal nitrides," Coord. Chem. Rev., vol. 257, no. 13-14, pp. 2015-2031, Jul. 2013.

[4] Y. Dong and J. Li, "Tungsten nitride nanocrystals on nitrogen-doped carbon black as efficient electrocatalysts for oxygen reduction reactions," Chem. Commun., vol. 51, no. 3, pp. 572-575, Jan. 2015.

[5] C. Giordano and M. Antonietti, "Synthesis of crystalline metal nitride and metal carbide nanostructures by sol-gel chemistry," Nano Today, vol. 6, no. 4, pp. 366-380, Aug. 2011.

[6] R. B. Levy and M. Boudart, "Platinum-like behavior of tungsten carbide in surface catalysis," Science (80. )., vol. 181, no. 4099, pp. 547-549, Aug. 1973.

[7] H. C. Park, S. J. Kim, M. C. Kim, D. M. Kim, and K. W. Park, "Tungsten nitride nanoplates as an anode material for lithium ion batteries," Ceram. Int., vol. 42, no. 1, pp. 1933-1942, Jan. 2016.

[8] R. Kieffer, P. Schwarzkopf, R. Kieffer, and P. Schwarzkopf, "Die Nitride," in Hartstoffe und Hartmetalle, Springer Vienna, 1953, pp. 206-250.

[9] D. H. Gregory, "Nitride chemistry of the s-block elements," Coord. Chem. Rev., vol. 215, no. 1, pp. 301-345, May 2001.

[10] H. A. Wriedt, “The N-W (nitrogen-tungsten) system,” Bull. Alloy Phase Diagrams, vol. 10, no. 4, pp. 358-367, Aug. 1989. 